\title{
Géneros y formación ingenieril: de la universidad a la industria
}

Gender and Engineering Education: From the University to the Industry Genres et formation en Ingénierie: de l'université à l'industrie Gêneros e formação da engenharia: da universidade para a indústria

Fecha de recepción: 30 DE SEPTIEMBRE DE 2013 / Fecha de aceptación: 6 DE MAYO DE 2015 / Fecha de disponibilidad en línea: 30 DE OCTUBRE DE 2015

Encuentre este artículo en http://magisinvestigacioneducacion.javeriana.edu.co/

\section{Resumen}

A partir del último tercio del siglo $X X$, ha crecido el interés por las producciones discursivas de la formación académica. El objetivo de este artículo de investigación es presentar el repertorio y la distribución de los géneros demandados durante el ciclo específico de una carrera de Ingeniería Industrial en una universidad argentina. Los análisis de frecuencias y de distribución muestran una tendencia a la incorporación gradual de tareas del contexto industrial. Se evidencia una preponderancia del saber práctico y un interés por la inmersión temprana del estudiante en el ámbito técnico-profesional. Asimismo, se identifican aspectos cuya revisión podría contribuir al mejoramiento de las prácticas de enseñanza.

Escrito por Daniela Stagnaro

Universidad Nacional de General Sarmiento, UNGS

Buenos Aires, Argentina

dstagnar@ungs.edu.ar

Lucía Natale

Universidad Nacional de General Sarmiento, UNGS

Buenos Aires, Argentina Inatale@ungs.edu.ar

\section{Palabras clave}

UP Educación superior; formación de ingenieros;

géneros profesionales

\section{Transferencia a la práctica}

La institución en la que se llevó a cabo el estudio cuenta con PRODEAC, Programa para el Desarrollo de Habilidades de la Lectura y la Escritura Académica a lo largo de la Carrera. Entre otras acciones, el PRODEAC indaga sobre las tareas escritas que se les solicitan a los estudiantes con distintos propósitos. Uno de ellos es el de sugerir a los responsables de las distintas carreras cursos de acción informados por la investigación. En este sentido, el estudio que aquí se presenta resulta útil para analizar la necesidad de cambios en la gradación de los géneros solicitados a los estudiantes a lo largo de la carrera.

Para citar este artículo / To cite this article / Pour citer cet article / Para citar este artigo

Stagnaro, D. \& Natale, L. (2015). Géneros y formación ingenieril: de la universidad a la industria. magis, Revista Internacional de Investigación en Educación, 8(16), 91-108. http://dx.doi.org/10.11144/Javeriana.m8-16.gfiu 


\section{Keywords}

UP Higher Education; Engineering

education; professional gender

\section{Mots clés}

UP éducation supérieure; formation d'ingénieurs; genres professionnels

\section{Abstract}

From the last third of the twentieth century, the interest towards the discursive produc tions of academic education has increased The aim of this paper is to present the repertory and gender distribution within a specific cycle of an Industrial Engineering degree in a university in Argentina. The analysis of frequency and distribution show a tendency towards a gradual incorporation of assignments in the industria context. The analysis evidences a preponderance of practical knowledge and an interest in the early immersion of students in the technical and professional field. Also, aspects that deserve review are identified to help improve the teaching practices.

\section{Transfer to practice}

The institution where the study was conducted has PRODEAC, a program for the Development of reading and academic writing skills. Among other actions, PRODEAC explores the written assignments that students are required to write with different purposes. One of them is to suggest active courses, informed by research, to those responsible for the various academic programs. In this sense, this study is useful to analyze the need for changes in the gradation of gender requested to students along their academic programs.

\section{Résumé}

A partir du dernier tiers du XXème siècle, l'intérêt pour les productions discursives dans la formation académique a crû. L'objectif de cet article de recherche est celui de présenter le répertoire et la distribution des genres demandés pendant le cycle spécifique d'une carrière d'Ingénierie Industrielle dans une université argentine. Les analyses de fréquences et de distribution montrent une tendance à l'incorporation graduelle de tâches du contexte industrielle. On a montré une prépondérance du savoir pratique et un intérêt pour l'immersion précoce de l'étudiant dans le domaine techniqueprofessionnel. De la même manière, on a identifié les aspects dont la révision pourrait contribuer dans l'amélioration des pratiques d'enseignement.

\section{Transfert à la pratique}

L'institution dans laquelle on a réalisé l'étude compte avec PRODEAC, Programme pour le Développement de Habilités de la lecture et I'Ecriture Académique tout au long de la carrière. Parmi d'autres actions, le PRODEAC indague par rapport aux devoirs écrits qu'on sollicite aux étudiants avec des propos différents. Un parmi d'eux est celui de conseiller aux responsables des différents carrières les cours d'action informés par la recherche. Ainsi, l'étude qu'on présente ici résulte utile pour analyser le besoin de changements dans la graduation des genres sollicités aux étudiants tout au long de leur carrière.

\section{Palavras-chave}

Ensino superior; engenharia; gêneros acadêmicos; gêneros profissionais

\section{Resumo}

Nas últimas décadas, tem havido um interesse crescente pelas produções discursivas da formação acadêmica. O objetivo deste artigo de pesquisa é apresentar o repertorio e distribuição em gêneros durante o ciclo específico do curso de Engenharia Industrial em uma universidade argentina. As análises de frequência e distribuição demostram uma tendência à incorporação gradual de tarefas do contexto industrial. Evidencia-se uma preponderância do saber prático e um interesse pela imersão precoce do discente no campo técnico-profissional. Mesmo assim, são identificados aspectos cuja revisão poderia contribuir para o meIhoramento das práticas de ensino.

\section{Transferência à prática}

A instituição na que foi realizado o estudo conta com PRODEAC, Programa para o Desenvolvimento de Habilidades da Leitura e a Escrita Acadêmica ao longo do Curso. Entre outras ações, o PRODEAC investiga sobre as tarefas escritas que Ihes são solicitados aos discentes com diferentes propósitos. Um deles é o de sugerir aos responsáveis dos diferentes cursos, linhas de ação informadas pela pesquisa. Neste sentido, o estudo que aqui é apresentado resulta útil para analisar a necessidade de mudanças na gradação dos géneros solicitados aos discentes ao longo do curso. 


\section{Introducción}

El crecimiento de la educación superior de las últimas décadas ha impulsado la incipiente conformación de la llamada alfabetización académica (Carlino, 2003) o alfabetizaciones académicas (Carlino, 2013; Lea \& Street, 1998). Se trata de un área interdisciplinaria, que involucra especialistas de diversas disciplinas, sobre todo de lingüística, educación y psicología.

En este contexto, las producciones escritas de los estudiantes han sido tomadas como objeto de estudio en distintas indagaciones que inicialmente se concentraron en las dificultades que los géneros propios de los estudios superiores generaban a los nuevos usuarios (por ejemplo, CasteIló, Bañales-Faz \& Vega-López, 2013; Giudice, Natale \& Stagnaro, 2008; Padilla, 2004, 2012; Sabaj, 2009). Por otro lado, se han desarrollado investigaciones dedicadas a la descripción de los géneros científicos y a los que se emplean durante la formación (entre muchos otros, Ciapuscio, 2000; Cubo de Severino, 2005; Gallardo, 2005; Natale, 2012; Navarro, 2014; Padilla, Douglas \& López, 2010). En esta línea, también se encuentran manuales de escritura destinados a estudiantes del nivel superior que parten de las secuencias textuales para el abordaje de los géneros (por ejemplo, Narvaja de Arnoux, Alvarado, Balmayor, di Stefano, Pereira \& Silvestri, 1998; Narvaja de Arnoux, di Stefano \& Pereira, 2002; Padilla, Douglas \& López, 2010; Pereira, 2005). Otros proyectos más recientes se han ocupado de relevar y analizar los repertorios o las familias de géneros que son solicitados a lo largo de distintas carreras (Gardner \& Nesi, 2012; Natale, 2013a; Stagnaro \& Martínez, 2013; Stagnaro \& Navarro, 2013). Más tardíamente y con menor fuerza, ha surgido la preocupación por el reconocimiento y la caracterización de los géneros profesionales (Montolío \& López-Samaniego, 2010; Parodi, 2008; Parodi, Ibáñez, Venegas \& González, 2010).

En lo que respecta a la formación de los estudiantes de ingeniería, hay una creciente conciencia sobre la necesidad de atender al desarrollo de sus competencias comunicativas. Se entiende hoy que el buen desempeño del ingeniero requiere el manejo de un amplio repertorio de géneros y el desarrollo de habilidades lingüísticas que le permitan interactuar tanto con operarios como con directores de empresas o clientes. En relación con esta necesidad, en Argentina, la Comisión Nacional de Evaluación y Acreditación Universitaria (CONEAU) ha impuesto como requisito para la aprobación de las carreras de ingeniería que consideren las competencias comunicativas en sus planes de estudio. Asimismo, en este país también se están implementando políticas educativas que apuntan a impulsar las carreras de ingeniería (MinCyT, 2011). Por estos motivos, los estudios tendientes a contribuir a la mejora del desarrollo de estas carreras adquieren una especial relevancia en la actualidad.

En este artículo, se presentan los resultados de la primera etapa de una investigación que estudia los géneros académicos y profesionales demandados durante la formación de grado de una carrera de ingeniería industrial de una universidad pública argentina. Esta investigación contempla tres perspectivas para la aproximación al estudio de los géneros: la de los docentes, la de los estudiantes — los sujetos participantes_ y la de los textos - el producto-. Aquí, se considera la perspectiva de los docentes recogida en entrevistas para explorar los géneros que se demandan en la formación académica del ingeniero industrial y analizar, luego, cómo se distribuyen a lo largo de la carrera. Interesa también observar las formas de vinculación con el conocimiento y la práctica profesional que son promovidas desde la universidad. Se espera que esta exploración aporte datos útiles para el diseño de intervenciones didácticas tendientes a favorecer el

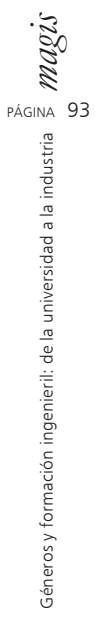

Descripción del artículo | Article description | Description de l'article | Artigo descrição

Este artículo de investigación se enmarca en el proyecto Prácticas de escritura profesional en contexto empresarial, que busca contribuir a la descripción de los géneros profesionales y brindar insumos para el diseño de propuestas didácticas en carreras de ingeniería. 
proceso de alfabetización disciplinar y a facilitar el tránsito entre la formación universitaria y la posterior inserción profesional en la industria.

\section{La enseñanza de la escritura en el nivel superior}

Desde mediados de la década de 1970, especialmente en Estados Unidos, en el marco del movimiento Writing Across the Curriculum (WAC), comenzaron a desarrollarse diversas iniciativas tendientes a promover las prácticas de lectura y escritura específicas del nivel superior (Russell, 1991). Latinoamérica no tardó en sumar proyectos con la misma finalidad, en un contexto de expansión de la matrícula universitaria impulsada por la democratización de los claustros. Propuestas como las desarrolladas en Argentina (Natale, 2013b), Colombia (Cisneros, Jiménez \& Rojas, 2010; González-Pinzón, 2012; Molina-Natera, 2012; Pérez-Abril \& RodríguezManzano, 2013) y otros países de la región (para un panorama general, Carlino, 2012) prueban la dimensión que ha adquirido la problemática, también abordada desde la investigación lingüística (Parodi, Ibáñez, Venegas \& González, 2010).

La consolidación del campo disciplinar se verifica en la creación de términos propios. Uno de ellos es el de alfabetización académica (Carlino, 2003, 2013) o alfabetizaciones académicas (Lea \& Street, 1998, 2006), que parece estar imponiéndose en la actualidad.

Las primeras definiciones de alfabetización académica hacían referencia a un conjunto de nociones, estrategias y prácticas lingüísticas y de pensamiento que un estudiante necesita haber desarrollado para aprender en la universidad y participar en las distintas culturas disciplinares (cfr. Carlino, 2003, p. 410).

Recientemente, Paula Carlino (2013) propuso una reformulación de la definición ${ }^{1}$ por ella elaborada diez años antes, en la que se nota un importante giro, ya que traslada el énfasis en los procesos de los estudiantes a las acciones que las propias instituciones y los docentes despliegan para favorecer el acceso del estudiante a las diferentes culturas disciplinares.

Por otro lado, desde el movimiento de Academic Literacies (ACLITS) (Lea \& Street, 1998; Lillis, 2003, entre otros), se subraya la idea del fenómeno de la alfabetización no como un proceso individual, de naturaleza psicológica o cognitiva, observable en "habilidades", sino como una práctica social, cultural, histórica e institucional, de lo que se deduce que la alfabetización no es universal ni unívoca, sino múltiple y socialmente situada.

La investigación sobre las prácticas de escritura en las disciplinas y su enseñanza ha sido abordada desde diversos encuadres teóricos. En el mundo anglosajón, han avanzado distintas corrientes: Inglés con Propósitos Académicos (IPA/EPA) (Swales, 1990), la Nueva Retórica (Bazerman, 1988; Berkenkotter \& Huckin, 1995, por ejemplo), la Teoría de Género y Registro (TGyR) de la Lingüística Sistémico Funcional (Rose \& Martin, 2012) y el Movimiento de las Alfabetizaciones Académicas (Lea \& Street, 1998;

1 En palabras de la autora: "alfabetización académica [hace referencia] al proceso de enseñanza que puede (o no) ponerse en marcha para favorecer el acceso de los estudiantes a las diferentes culturas escritas de las disciplinas. Es el intento denodado por incluirlos en sus prácticas letradas, las acciones que han de realizar los profesores, con apoyo institucional, para que los universitarios aprendan a exponer, argumentar, resumir, buscar información, jerarquizarla, ponerla en relación, valorar razonamientos, debatir, etcétera, según los modos típicos de hacerlo en cada materia. Conlleva dos objetivos que, si bien relacionados, conviene distinguir: enseñar a participar en los géneros propios de un campo del saber y enseñar las prácticas de estudio adecuadas para aprender en él. En el primer caso, se trata de formar para escribir y leer como lo hacen los especialistas; en el segundo, de enseñar a leer y a escribir para apropiarse del conocimiento producido por ellos" (Carlino, 2013, p. 370). 
Lillis \& Scott (2007). Todas ellas toman como punto de partida la noción de género, tanto para la descripción y la clasificación de los textos que los estudiantes deben leer y producir como para el establecimiento de vínculos entre los géneros y los contextos sociales o las culturas disciplinares (Hyland, 2000) en los que circulan.

En términos generales, en estas corrientes se reconocen dos dimensiones del género unidas de manera inextricable. Por un lado, los géneros son conceptualizados como modos de realizar un tipo de acción particular en un contexto social o ámbito específico de actividad. Por el otro, se destaca el rol que el lenguaje y otros sistemas semióticos juegan en el cumplimiento de la acción. En este sentido, los géneros pueden ser analizados desde su dimensión lingüística, en la que se reconocen distintos planos, desde los más ligados a la situación comunicativa, como el registro (Halliday, 1978), hasta su manifestación en los recursos léxicos y gramaticales y en su organización discursiva.

\section{Propuestas de clasificación de géneros académico-estudiantiles}

La clasificación de las producciones requeridas a los estudiantes durante su formación aparece como una de las preocupaciones centrales en numerosas investigaciones recientes. Un tipo de agrupamiento parte de considerar la existencia de dos polos o extremos, uno propio de la vida universitaria y otro, de las culturas disciplinares y profesionales.

Susan Peck MacDonald (1994) propone un esquema que contempla la evolución de las escrituras desde una redacción personal hasta la adopción de un registro puramente disciplinar, propio de la prosa de un experto. El esquema consta de diversas fases, que se vinculan con el grado de avance en los estudios. En un primer momento o estadio, en los últimos años de la escuela media, los estudiantes suelen producir textos sobre experiencias personales o ensayos breves sobre un tema. Ya en la universidad, se los introduce en las generalidades de la escritura académica. Y recién al final de sus trayectorias formativas comienza a exigirse una prosa con rasgos acordes a los de la comunidad disciplinar experta.

Giovanni Parodi, Romualdo Ibáñez, René Venegas y Cristian González (2010) plantean un esquema bipartito, en el que se distinguen los géneros académicos y los profesionales, que se enmarcan en un tipo de discurso especializado en particular. En un estudio empírico, realizado en una universidad chilena, relevan los textos que los estudiantes leen en cuatro carreras de grado (ingeniería en construcción, química industrial, trabajo social y psicología) y los empleados en la comunicación escrita de los ámbitos profesionales correspondientes e identifican un total de veintinueve géneros, de los cuales solo uno pertenece exclusivamente al ámbito académico (Guía Didáctica); ocho son empleados en ambos ámbitos y veinte en el ejercicio profesional. De acuerdo con estos resultados, por un lado, establecen un continuum en el acceso y la incorporación en las comunidades disciplinares, verificable en la intersección de ambos grupos, aunque tal continuidad no es necesariamente el efecto de una planificación intencional (Parodi, Ibáñez, Venegas \& González, 2010). Por otro, remarcan que la vida laboral exige el manejo de una mayor variedad de géneros con una amplia diversidad de propósitos comunicativos, organización discursiva y rasgos léxico-gramaticales, pero que esta multiplicidad no se ve reflejada en los géneros requeridos durante la formación universitaria. En este sentido, las diferencias entre los textos propios de uno y otro ámbito resultan un "escollo en el adecuado transitar desde la academia al medio laboral" 
y pueden "ciertamente, constituirse en una lentificación del proceso de integración" que "afectaría al desempeño inicial del sujeto ya graduado" (Parodi, Ibáñez, Venegas \& González, 2010, p. 286).

En la misma línea, Daniel Cassany y Carmen López-Ferrero (2010) contrastan el tipo de escritos que son solicitados a los estudiantes durante su formación de grado y los que deben producir al momento de su inserción laboral y llegan a conclusiones similares que los investigadores chilenos: encuentran que las prácticas discursivas del ámbito profesional son diferentes de las académicas "no solo en los tipos de textos y en sus rasgos estilísticos, sino sobre todo en sus parámetros pragmáticos y en los procesos de producción y recepción, que suelen involucrar a más personas, idiomas y tareas" (p. 369). Señalan, además, que las prácticas letradas profesionales suelen ser más dinámicas y cambiantes que las que se proponen en la universidad, debido a que incluyen no solo el modo escrito en su versión tradicional, sino también el empleo de variados soportes y lenguajes.

Otro tipo de clasificación es la que elaboran Sheena Gardner y Hilary Nesi (2012), a partir del análisis de un extenso corpus, compuesto por 2.858 textos producidos por estudiantes de más de treinta carreras. Proponen un esquema que contempla, ante todo, las metas genéricas y los pasos que conforman los géneros relevados, retomando criterios propios de la tradición de la Lingüística Sistémico Funcional (LSF) y los lineamientos pedagógicos de la Escuela de Sydney (Rose \& Martin, 2012). Agrupan los textos en trece familias: estudio de caso, crítica, diseño, escritura empática, ensayo, ejercicio, explicación, relevamiento bibliográfico, metodología, recuento narrativo, resolución de una situación problemática, propuesta e informe de investigación².

Los estudios de caso buscan desarrollar/demostrar la comprensión de las prácticas profesionales a partir del análisis de un caso particular, por lo general, complejo. Para ello, se requiere la descripción del caso, el análisis y las sugerencias para acciones futuras. Se trata de un género típicamente profesional utilizado en diversas áreas (negocios, medicina, ingeniería).

Las críticas tienen por objeto desarrollar/demostrar la comprensión de un objeto de estudio y la capacidad para evaluar su importancia. Incluyen la descripción del objeto y un segmento evaluativo. Abarcan un amplio espectro de objetos, desde la crítica de un libro hasta la evaluación de un proyecto, una organización, un sector de la economía o un producto industrial. Corresponden tanto a la actividad profesional como a la formativa.

Los diseños procuran desarrollar/demostrar la habilidad de diseñar un producto o un procedimiento que puede ser luego manufacturado o implementado, según sea el caso. Se componen de uno o varios objetivos, el desarrollo del diseño y la evaluación de su puesta en práctica. Circulan en ámbitos profesionales y académicos.

La familia denominada escritura empática agrupa distintos géneros que buscan demostrar y desarrollar la capacidad de "traducir" cuestiones académicas a otros ámbitos no expertos. Se incluyen en ella consejos de expertos para la industria, artículos de divulgación y también otros géneros privados, como aplicaciones a empleos y hojas de vida.

Los ensayos tienen como propósito desarrollar/demostrar la habilidad para construir una argumentación coherente y emplear el pensamiento crítico a partir de una cuestión. Se componen de tres pasos: introducción, argumentos y una conclusión. Son comunes en la vida universitaria y

2 La traducción de los nombres de los géneros es nuestra. Las etiquetas propuestas por las autoras, en inglés, son las siguientes: Case Study, Critique, Design Specification, Empathy Writing, Essay, Exercise, Explanation, Literature Survey, Methodology Recount, Narrative Recount, Problem Question, Proposal y Research Report. 
también suelen encontrarse entre los textos publicados por especialistas.

Los ejercicios tienen como meta servir como práctica de habilidades básicas (cálculos, definiciones de términos técnicos, análisis de datos o procedimientos elementales) para consolidar conocimientos clave de las disciplinas.

Las explicaciones apuntan al desarrollo y a la demostración de la comprensión de un objeto de estudio. Se componen de una descripción y una explicación. Pueden realizarse como textos expertos completos, o bien en partes de un informe de investigación o de una crítica.

Los relevamientos bibliográficos buscan desarrollar/demostrar el conocimiento de la literatura sobre un tema de estudio. Se componen de una síntesis del texto leído y de una evaluación crítica. Entre los géneros que se incluyen en esta familia se hallan, por ejemplo, la reseña y el estado del arte. Circulan tanto en el ámbito académico como en el experto, bajo la forma de artículos.

La metodología permite desarrollar/demostrar cierta familiaridad con los procedimientos, métodos y convenciones disciplinares necesarios para el registro de los hallazgos experimentales o empíricos. Entre los géneros comprendidos en esta familia se encuentran, entre otros, los informes de laboratorio, de campo y forenses.

Las narrativas tienen como finalidad potenciar la conciencia sobre las motivaciones y comportamientos de individuos y de organizaciones. En esta familia se encuentran diversos géneros, como la autobiografía, las etnografías, los informes sobre accidentes. Se incluyen en esta clase las memorias de las empresas, en las que se presentan sus orígenes y su evolución, su misión, sus objetivos, entre otros datos relevantes para su caracterización.

La familia de los problemas o situaciones problemáticas procura proveer de práctica para la aplicación de métodos en respuesta a problemas profesionales a partir de casos que simulan situaciones reales. Los pasos que componen los géneros de esta familia (análisis de problemas legales, escenarios comerciales o casos médicos, por ejemplo) son el problema, los argumentos y una posible solución. Se trata, entonces, de géneros de formación que guardan similitudes con tareas profesionales.

Las propuestas permiten desarrollar/demostrar habilidades para organizar un curso de acción a partir de un propósito determinado. Forman parte de esta familia los planes de negocio, los proyectos de investigación, los planes de reforma legislativa, los proyectos industriales y los de mejora. Se componen de un propósito, un plan detallado basado en argumentos. Circulan en ámbitos profesionales y académicos.
Finalmente, los informes de investigación buscan desarrollar/demostrar la capacidad de llevar adelante una investigación, lo que incluye su diseño, ejecución y apreciación de las contribuciones a un campo de estudio. La familia incluye tanto los proyectos estudiantiles como los artículos de investigación publicados.

Para los fines de este estudio, esta última clasificación resulta sumamente productiva para organizar y analizar las demandas que realizan los docentes a los estudiantes del último tramo de la carrera de ingeniería industrial.

\section{La institución}

La Universidad Nacional de General Sarmiento (UNGS) se encuentra ubicada a unos $30 \mathrm{~km}$ de Buenos Aires, ciudad capital de Argentina. Fue fundada en 1993. Asisten a ella cerca de 5.315 estudiantes, es decir, un 0,4\% (SPU, 2010) del total de la población argentina que cursa estudios universitarios en instituciones públicas, por lo que puede considerarse una institución relativamente pequeña.

En cuanto al perfil del alumnado, la mayoría proviene de hogares de trabajadores, que podrían encuadrarse en grupos socialmente desfavorecidos o de clase media baja. Según algunos estudios iniciales (Gentile \& Merlinsky, 2003), el 55\% de los padres de los estudiantes solo había alcanzado a completar estudios primarios y alrededor del 30\% completó los secundarios. Investigaciones recientes señalan que el $81,2 \%$ es primera generación en educación superior (Ezcurra, 2011, p. 49). De esta caracterización de la población estudiantil puede inferirse un escaso contacto con materiales escritos de características similares a los empleados en el nivel superior, al menos en el seno del hogar.

Dada esta situación y con base en distintos diagnósticos sobre las competencias cognitivas y discursivas realizados en los años en que la Universidad iniciaba sus actividades, desde sus documentos fundacionales se contempló la necesidad de afianzar el dominio de la lectura y la escritura. Con esta finalidad, la UNGS implementó dos talleres sobre habilidades generales (Carlino, 2013), destinados a ingresantes y un programa para la enseñanza de los textos propios de las distintas disciplinas: el Programa para el Desarrollo de Habilidades de la Lectura y la Escritura Académica a lo largo de la Carrera (PRODEAC). Se trata de un programa institucional que procura apoyar los procesos de alfabetización disciplinar a lo largo de la formación de grado, para lo que participa del dictado de asignaturas de diversas carreras. Las clases en las que se trata la producción de textos son impartidas por una pareja pedagógica formada por un especialista en el área disciplinar y un docente de 
lectura y escritura. En otras palabras, es una propuesta de enseñanza de la escritura basada en géneros, interdisciplinaria, curricular, sistemática y gradual, que se nutre de la investigación (Natale \& Stagnaro, 2012). Una de las tareas de este programa es la de brindar asesoramiento a los coordinadores de carrera y a los docentes sobre los textos que se exigen en las asignaturas, para lo que previamente se indagan los planes de estudio. En este contexto, este estudio adquiere relevancia.

\section{La carrera de ingeniería industrial}

La estructura curricular de ingeniería industrial se organiza en tres tramos ciclados. En primer lugar, un Curso de Aprestamiento Universitario (CAU) común a todas las carreras de la UNGS, en el que se abordan, fundamentalmente, competencias generales de lectocomprensión y matemática.

En segundo lugar, un Primer Ciclo Universitario (PCU) con un grupo de materias comunes a las ingenierías y las carreras afines, con una fuerte base de matemáticas, física, química y tecnologías básicas, como mecánica de fluidos o termodinámica. EI PCU tiene una duración de cinco semestres durante los que se cursan diecinueve asignaturas y se obtiene el diploma universitario de estudios generales con mención en tecnología industrial.

Finalmente, la estructura curricular se completa con el Segundo Ciclo Universitario (SCU) en el que se encuentran las asignaturas más específicas del campo disciplinar. Este ciclo cuenta con veintiocho asignaturas.

Según se detalla en la página web de la UNGS (www.ungs.edu.ar/), el título de ingeniero industrial capacita y habilita para el desempeño en el ámbito industrial y la realización de diversos tipos de tareas vinculadas, principalmente, con el estudio, la planificación y la programación, el diseño, la dirección, la administración de recursos, el seguimiento y la evaluación, y la mejora continua de los procesos y las plantas.

\section{Metodología}

Como se señaló en la introducción, aquí se presentan los resultados de la primera etapa de una investigación mayor. Esta fase inicial consistió en una exploración etnográfica de los géneros, basada en entrevistas a los docentes de una carrera de ingeniería industrial. Interesa particularmente su perspectiva, debido al rol que ocupan en el proceso de formación de los futuros miembros de la comunidad disciplinar. De acuerdo con la propuesta de Daniel Cassany y Carmen López-Ferrero (2010), esta primera aproximación etnográfica servirá de base para realizar futuros contrastes con análisis textuales. En otras palabras, los resultados aquí presentados no contemplan el análisis de un corpus textual, sino de entrevistas.

Este estudio se focaliza en el SCU de la carrera de ingeniería industrial compuesto por las materias troncales, es decir, aquellas que se relacionan directamente con las incumbencias del ingeniero industrial y lo diferencian de cualquier otro tipo de ingeniero.

Siguiendo a Sheena Gardner y Laura Powell (2006), el relevamiento de información acerca de los géneros demandados durante el curso de las materias se efectuó a partir del análisis de entrevistas. Se realizó una entrevista semiestructurada a cada uno de los veintiséis profesores que conforman el cuerpo docente de las asignaturas específicas, es decir, el universo completo de los profesores de la carrera que se tomó como caso de estudio ${ }^{3}$. Los docentes entrevistados son docentes regulares de la universidad, mayormente ingenieros sin una formación específica en docencia universitaria, aunque con trayectoria en el ámbito profesional.

El objeto de las entrevistas fue la indagación acerca del tipo de tareas demandadas a los estudiantes en instancias de evaluación parcial. Se les pidió a los profesores información detallada sobre las tareas, sus metas y el tipo de producto esperado. En algunos casos, las respuestas fueron precisas, mientras que en otros se formularon con vaguedad, por lo que fue necesario repreguntar $u$ ofrecer opciones para que pudieran identificar las metas perseguidas por la tarea solicitada.

Una vez tomadas las entrevistas, estas fueron analizadas a partir de las categorías propuestas por Gardner y Nesi (2012), presentadas en la sección previa. Para la determinación de las familias de géneros, se consideró como indicador central la meta explicitada por el docente y se la cotejó con las caracterizaciones de las autoras. Cuando los datos recogidos de las entrevistas no resultaban suficientes para establecer la clasificación, se requirió un encuentro complementario con el docente en el que se le pidió la ampliación de la información aportada.

Posteriormente, se analizaron cuantitativamente los datos: se midieron las frecuencias y la distribución de las familias de géneros a lo largo de SCU. Luego, se analizaron cualitativamente los datos y se interpretaron los resultados.

Finalmente, cabe aclarar que no se pretende hacer generalizaciones sobre las carreras de ingeniería, sino aportar datos sobre la frecuencia y distribución de los géneros en una carrera en particular. Sí se busca que los datos promuevan la reflexión sobre los géneros que se solicitarán a los estudiantes en otras carreras y su secuenciación y, en última instancia, que sirvan

3 Se exceptuaron los dos niveles de idioma inglés, debido a que toman un objeto de enseñanza diferente de los contenidos técnicos. 
como insumo a la hora de diseñar intervenciones didácticas que favorezcan la alfabetización disciplinar.

\section{Análisis de frecuencia de los géneros en el SCU}

Los resultados obtenidos a partir de las entrevistas se sintetizan en el gráfico 1. En él se exponen las frecuencias de todas las familias de géneros, considerando como base el total de ocurrencias a lo largo de todo el SCU de ingeniería industrial.

Los datos muestran que la familia del problema, es decir, aquella que presenta a los estudiantes situaciones problemáticas propias de empresas e industrias aparece como la más requerida (37,11\%). Estos escenarios simulados obligan al estudiante a buscar soluciones posibles, poniendo en juego los contenidos técnicos desarrollados durante las clases. Según lo manifestado por un docente en una entrevista, estos trabajos interesan particularmente porque habilitan la posibilidad de que se propongan distintas soluciones, todas viables. Aquí no cuenta la memorización de los contenidos, sino la búsqueda de soluciones para una situación. En un estudio sobre tareas de escritura en distintas carreras y en distintos países, Marilyn Lewis y Donna Starks (1997) hallaron que las situaciones problemáticas son más frecuentes en la formación técnico-profesional. En este sentido, estos resultados coinciden con los encontrados en otras latitudes.

Gráfico 1

Porcentajes de ocurrencia de las familias en el SCU de ingeniería industrial

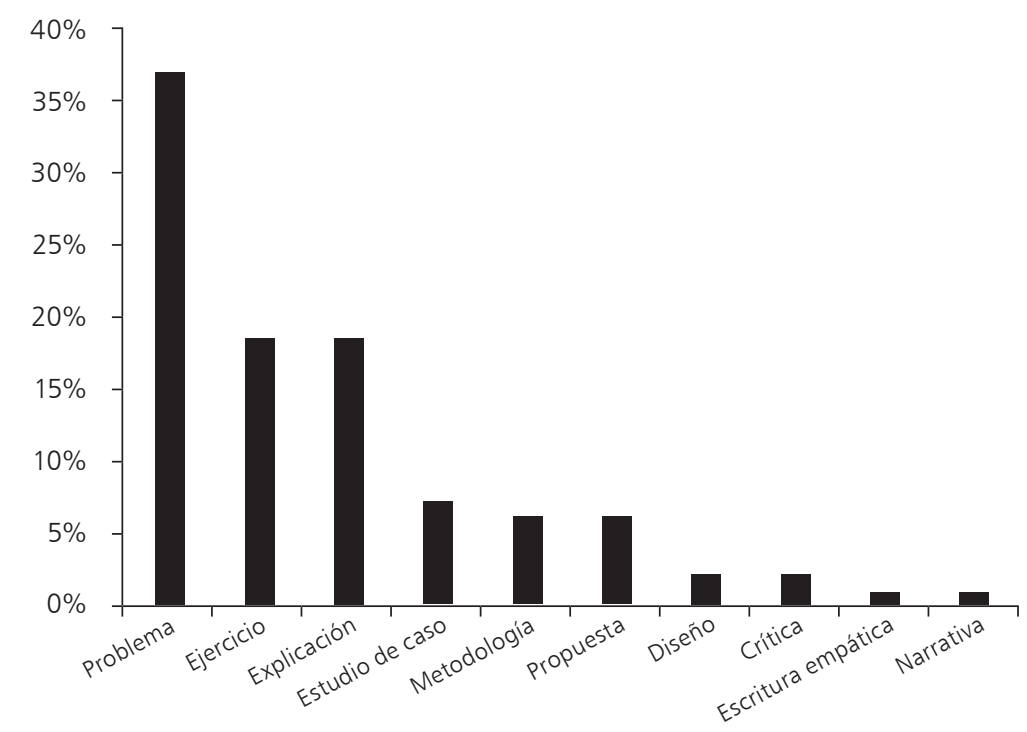

Fuente: elaboración propia

Siguen en orden de frecuencia los ejercicios $(18,56 \%)$ y las explicaciones (18,56\%). De acuerdo con la caracterización antes propuesta, estas dos familias conforman un grupo que tiene como objetivo principal promover el estudio de conocimientos básicos de la disciplina, como fórmulas matemáticas o conceptos y la descripción de sus objetos de estudio. En este sentido, constituyen las dos familias más directamente ligadas a la formación inicial, más centrada en lo académico, que aún no involucra desafíos del mundo profesional. 
El relevamiento realizado muestra que los ejercicios que se solicitan en esta carrera en particular responden a dos tipos principales. Por un lado, se encuentran los ejercicios de aplicación, los más frecuentes, que demandan la resolución de un cálculo; por el otro, aparecen, aunque en menor medida, los que exigen el conocimiento de conceptos teóricos. En dos casos se detectó una variante, que solo implica la identificación de definiciones en exámenes de opciones múltiples. Según los docentes que los utilizan, esta forma de evaluación les permite "controlar que los estudiantes estudien".

Siguiendo la caracterización de los ejercicios propuesta, los problemas aparecen como formas más complejas y más elaboradas que los ejercicios que, si bien implican resolver un cálculo, no presentan, en principio, la posibilidad de que haya más de una solución posible. Otra diferencia radica en que los cálculos demandados no suelen ser puestos en relación con un contexto concreto en particular.

Las metodologías $(6,19 \%)$ aparecen también ligadas a la simulación de situaciones profesionales. En su mayoría, son informes de laboratorio, género que desafía a los estudiantes a poner en práctica conocimientos adquiridos previamente. En función de la descripción de una de las docentes, en este tipo de prácticas:

[...] de acuerdo a los ejercicios que hicimos en clase y a los ejercicios que ellos tienen para desarrollar, lideramos una práctica de laboratorio. Ahí se hacen ejercicios similares, con aplicaciones similares a las que vimos, pero están en la guía para que ellos lo implementen en la realidad y puedan tomar las mediciones, ver cómo funcionan, ver las curvas y puedan comparar si están viendo lo mismo que vieron en la clase [...] Ellos hacen el montaje del circuito, toman las mediciones, desarrollan las preguntas que hacen. Toman los datos, las fotos, las imágenes, y tienen 15 días después para enviar el informe.

Los datos arrojados por el gráfico 1 también permiten advertir que un porcentaje interesante (55\%) de las producciones solicitadas a los estudiantes tiene como escenario imaginario o real la industria o el ámbito laboral. A su vez, el 18\% de estos géneros pone en contacto directo y efectivo al estudiante con una empresa o industria existente (estudios de caso, diseños y propuestas y narrativa), e incluso lo desafían a revisar sus propios intereses y a proyectarse en ese ámbito.

Finalmente, se advierte la ausencia de ciertas familias de géneros en el SCU de ingeniería industrial, concretamente, las del ensayo y el relevamiento bibliográfico. Esto evidencia la preponderancia del interés por un saber práctico. Parece que el plantel docente tiene el foco de sus preocupaciones en el entrenamiento de los estudiantes en el saber hacer, el saber aplicar antes que en el saber interpretar y comunicar lo que dicen los textos teóricos.

\section{Análisis de distribución de los géneros a lo largo del SCU}

Una vez observada la frecuencia de la solicitud de los géneros pertenecientes a las distintas familias, se ha atendido a su distribución a lo largo de todo el SCU para indagar, por un lado, si hay una progresión en relación con el grado de complejidad de las demandas a medida que se avanza en la estructura curricular; y, por otro, las formas de vinculación con el conocimiento que se promueven a lo largo de la carrera. La tabla 1 sintetiza de manera gráfica la información relevada. 
Tabla 1

Distribución de las familias de géneros según los semestres de la carrera

\begin{tabular}{l|c|c|c|c|c|c}
\hline & \multicolumn{6}{c}{ Semestres de la carrera } \\
\hline Familias de géneros & $\mathbf{6}^{\mathbf{0}}$ & $\mathbf{7 0}^{\circ}$ & $\mathbf{8}^{\circ}$ & $\mathbf{9}^{\circ}$ & $\mathbf{1 0}$ & Totales \\
\hline Problema & 3 & 11 & 11 & 8 & 3 & 36 \\
\hline Ejercicio & 7 & 4 & 4 & 1 & 2 & 18 \\
\hline Explicación & 2 & 2 & 2 & 6 & 6 & 18 \\
\hline Estudio de caso & 0 & 0 & 0 & 1 & 6 & 7 \\
\hline Metodología & 3 & 2 & 0 & 0 & 1 & 6 \\
\hline Propuesta & 0 & 2 & 0 & 3 & 1 & 6 \\
\hline Diseño & 0 & 2 & 0 & 0 & 0 & 2 \\
\hline Crítica & 0 & 0 & 0 & 1 & 1 & 2 \\
\hline Escritura empática & 0 & 0 & 0 & 1 & 0 & 1 \\
\hline Narrativa & 1 & 0 & 0 & 0 & 0 & 1 \\
\hline Totales & 16 & 23 & 17 & 21 & 20 & 97 \\
\hline \hline
\end{tabular}

Fuente: elaboración propia

El promedio de trabajos solicitados por semestre es de 19,4. Los semestres con menores demandas son el sexto (16) y el octavo (17). El semestre que registra mayor número de demandas es el séptimo.

La cantidad de trabajos requeridos puede ser puesta en relación con el grado de exigencia que las tareas imponen. En el sexto y el octavo semestre solo se piden géneros propios de la formación académica, de resolución relativamente sencilla, como ejercicios, explicaciones y problemas. En el séptimo, en cambio, aparece otro tipo de exigencia. Si bien el 74\% de los trabajos corresponde a las tareas más simples, el 26\% involucra géneros que exigen la aplicación de los conocimientos técnicos, como la propuesta y el diseño. Esto muestra que ya en el séptimo semestre el estudiante debe resolver tareas propias de la actividad profesional como el estudio de mercado o el diseño de un producto en la asignatura desarrollo de producto ${ }^{4}$. Por su parte, en investigación operativa, otra materia troncal ubicada en este período, los estudiantes deben ponerse en contacto con una empresa para analizar el sistema de gestión de stock. A partir de esto, deben realizar una propuesta de mejora y defenderla con pruebas cuantitativas concretas. Estos primeros trabajos centrados en la tarea profesional suelen ser retomados por los estudiantes en el décimo semestre, cuando inician las prácticas profesionales supervisadas. Se trata, entonces, de instancias que constituyen un hito en la formación que funcionan como referentes a la hora de planificar y decidir la práctica profesional supervisada.

Con respecto a la familia de la crítica, se registran solo dos ocurrencias y ambas corresponden a materias del dominio de la economía: estructura económica argentina y principios de economía. El hecho de que estos géneros no se identifiquen en asignaturas más específicas de la ingeniería y que los dos casos hallados correspondan al mismo dominio disciplinar podría estar señalando formas discursivas propias de esa otra comunidad disciplinar, con puntos de intersección con la comunidad ingenieril.

4 Se trata de una materia innovadora dentro del currículo de ingeniería industrial de Argentina. En ese espacio, los estudiantes deben crear un producto que no existe en el mercado y que podría cubrir una necesidad o generar una innovación en el mercado. 
Los géneros de familias propias del ejercicio profesional son solicitados en la última etapa del SCU. Las familias del estudio de caso, la propuesta, el diseño y la escritura empática tienen como espacio protagónico la industria, es decir, llevan al estudiante al ámbito laboral y lo obligan a observar situaciones, a proponer mejoras e, incluso, a desarrollar un producto y a proyectarse en este ámbito. El caso de la escritura empática se registra en el noveno semestre y la solicitud corresponde al seminario denominado entrevistas profesionales, en el que se les pide a los estudiantes la producción de un texto escrito en el que tienen que definir sus objetivos laborales y proyectarse en el ámbito tomando conciencia de sus fortalezas y debilidades, asimismo, se les requiere hacer una búsqueda laboral y diseñar su curriculum vitae.

Se ha detectado que las materias en las que aparecen las demandas de estas familias son las que — según el coordinador de la carrera- determinan las especificidades de ingeniería industrial, esto es, las que brindan los saberes definidos en las incumbencias: desarrollo de producto; investigación operativa; organización de la producción I, II, III; proyecto industrial; higiene, seguridad industrial y medio ambiente; práctica profesional supervisada.

\section{Complejidad creciente de los géneros}

El análisis de la distribución de los géneros a lo largo del SCU permite reconocer una gradación en las tareas que se solicitan. Se advierte que salvo el octavo semestre - en el que solo se registra el pedido de géneros propios del ámbito académico (problemas, ejercicios y explicaciones) — si se sigue la estructura curricular, se observa que semestre a semestre se evidencia un leve incremento de la demanda de tareas más cercanas al ámbito profesional. En el sexto, estas tareas representan el 25\%; en el séptimo, el $26 \%$; en el noveno, el $28,57 \%$ y en el décimo ascienden al $45 \%$. Estos datos evidencian un avance progresivo hacia los géneros profesionales, más recurrentes en el último semestre.

De esta manera, las situaciones hipotéticas o simuladas constituyen un primer acercamiento del estudiante al contexto industrial en los primeros semestres. Luego, se continúa este camino evolutivo con las familias de géneros más próximos al ámbito profesional: el estudio de caso y la propuesta, que implican la inmersión del estudiante en el contexto laboral real. Así, se incrementa, por un lado, el rol protagónico del contexto y, por el otro, la dificultad de las tareas demandadas, ya que en los estudios de caso y en las propuestas las problemáticas ya no son planteadas por los profesores, sino que deben ser detectadas y definidas por los estudiantes, quienes son puestos en la situación de sugerir soluciones.

Este camino evolutivo puede representarse gráficamente del siguiente modo.

Gráfico 2

Camino evolutivo de los géneros en la formación del ingeniero industrial

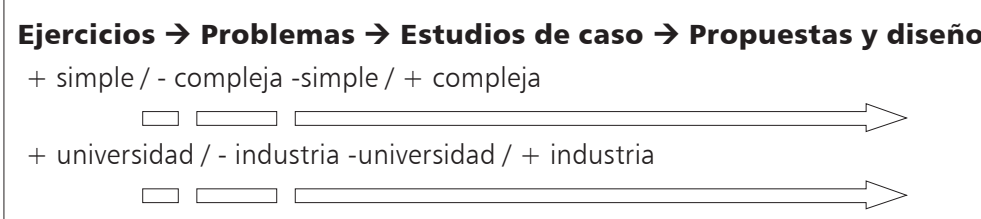

Fuente: elaboración propia 
Tal como muestra el gráfico 2, las demandas de los géneros aparecen organizadas en un continuum: el grado de cercanía con las tareas profesionales del ingeniero industrial se incrementa a medida que se avanza en la estructura curricular de la carrera. Esto implica que de tareas descontextualizas, típicas de una formación estudiantil tradicional, se va pasando progresivamente a tareas cada vez más fuertemente situadas en el ámbito industrial. De esta manera, se ponen en juego dos escalas: la complejidad y la contextualización. Este paso de tareas descontextualizadas a tareas contextualizadas podría estar funcionando como un factor que podría facilitar la inserción del graduado en el ámbito laboral.

\section{Conclusiones}

En el marco del incipiente desarrollo de los estudios sobre alfabetización profesional, este trabajo aporta información empírica sobre el ciclo específico de una carrera de ingeniería en Argentina. Una primera contribución es el mapeo completo de los géneros demandados en este ciclo de una carrera, lo que brinda una visión integral de las producciones solicitadas a los estudiantes en los distintos semestres de la estructura curricular. Estudios previos presentan información sobre los géneros más demandados, como el parcial, el informe o la monografía (por ejemplo, Ciapuscio, 2000; Gallardo, 2005), pero aún no se cuenta con los repertorios propios de cada disciplina, por lo que los resultados aquí presentados constituyen un aporte para el avance del conocimiento en esta línea.

Si bien se ha encontrado un predominio del interés por un saber práctico, parece que el foco de las preocupaciones del plantel docente está puesto en el entrenamiento de los estudiantes en el saber hacer, el saber aplicar y en la inmersión del estudiante en el ámbito técnicoprofesional antes que en el saber interpretar y comunicar lo que dicen los textos disciplinares, lo que podría evidenciar aspectos propios de la epistemología de la disciplina y de la formación propia del cuerpo docente.

Estos hallazgos coinciden con las observaciones realizadas por Cassany y López (2010, p. 369), quienes advierten que si bien durante la formación de grado se demandan actividades y géneros vinculados con lo profesional, estos no dejan de ser simulaciones controladas. Sin embargo, el estudio de corpus realizado por Giovanni Parodi, Romualdo Ibáñez, René Venegas y Cristian González (2010, p. 287) sugiere que la inclusión de este tipo de actividades puede deberse a la búsqueda de medios que sirvan para la paulatina integración de los estudiantes en su comunidad disciplinar.

Una segunda contribución del trabajo es la perspectiva desde la que se aborda la alfabetización en el ámbito superior, dado que, en general, predominan las miradas sobre las dificultades de los estudiantes para resolver las tareas (Riestra, 2002, 2008; Rinaudo, Donolo \& Paoloni, 2003; Rosales \& Vázquez, 2006). En este caso, se ha presentado una visión de la alfabetización académica que pone el énfasis en otro de los actores del proceso de enseñanza-aprendizaje: el docente y las demandas que realiza a los estudiantes durante los cursos de las materias. Estas tareas fueron reinterpretadas en términos de géneros como instrumentos mediadores del aprendizaje y a partir de allí, es posible inferir el sujeto de aprendizaje que se configura. En este sentido, se ha advertido un fuerte interés en el desarrollo de conocimientos técnicos y su aplicación práctica a situaciones problemáticas hipotéticas y reales del ámbito industrial que exigen la puesta en juego de los saberes disciplinares y la capacidad para identificar problemas y proponer mejoras. 
Los resultados, si bien no son generalizables a todas las carreras de ingeniería, revisten algunas implicaciones pedagógicas. Señalan algunos aspectos a ser considerados en la planificación de los géneros a incluir en las materias a lo largo de las carreras en función del sujeto de la educación y del profesional que la sociedad actual demanda: fundamentalmente, la intercalación tanto de géneros académicos como profesionales, su secuenciación en función de su grado de complejidad y la atención a la variedad de códigos disponibles.

Finalmente, coincidimos con Giovanni Parodi, Romualdo Ibáñez, René Venegas y Cristian González (2010, p. 249) en la necesidad de hacer avanzar el campo de la alfabetización disciplinar, a partir de la descripción de los textos que circulan en los ámbitos de especialidad. Así, la próxima etapa de la investigación prevé avanzar en las descripciones lingüísticas de los géneros relevados y en el relevamiento de los géneros del ámbito profesional del ingeniero industrial para ampliar el repertorio y disponer de insumos que permitan generar intervenciones didácticas más ricas y mejorar el proceso de alfabetización disciplinar y la inserción de los estudiantes en el ámbito laboral.

\section{Sobre las autoras}

Daniela Stagnaro es profesora y licenciada en letras, Universidad de Buenos Aires (UBA). Obtuvo una beca de la Agencia Nacional de Promoción Científica y Tecnológica y, actualmente, está desarrollando su tesis de doctorado. Realiza investigación y docencia vinculadas con el discurso académico-científico y profesional en el Instituto de Industria de la Universidad Nacional de General Sarmiento (UNGS).

Lucía Natale es profesora en letras, Universidad de Buenos Aires (UBA). Está finalizando la redacción de su tesis doctoral. Investiga sobre cuestiones vinculadas a la escritura académica y profesional. Coordina PRODEAC, Programa para el Desarrollo de la Lectura y la Escritura Académica a lo largo de la Carrera en la Universidad Nacional de General Sarmiento (Argentina).

\section{Referencias}

Bazerman, C. (1988). Shaping Written Knowledge. The Genre and Activity of the Experimental Article in Science. Madison, Wisconsin: The University of Wisconsin Press. Disponible en: http://wac.colostate.edu/ books/bazerman_shaping/shaping.pdf

Berkenkotter, C. \& Huckin, T. (1995). Genre Knowledge in Disciplinary Communication: Cognition/Culture/Power. New Jersey: Lawrence Erlbaum Associates, Inc.

Carlino, P. (2003). Alfabetización académica: un cambio necesario, algunas alternativas posibles. Educere. Revista Venezolana de Educación, 6 (20), 409-420. Disponible en: http://www.redalyc.org/pdf/ 356/35662008.pdf

Carlino, P. (2012). Who Takes Care of Writing in Latin American and Spanish Universities? En Chris Thaiss, Gerd Bräuer, Paula Carlino, Lisa Ganobcsik-Williams \& Aparna Sinha (eds.) (2012). Writing Programs Worldwide: Profiles of Academic Writing in Many Places, 485-498. Anderson, South Carolina: Parlor Press. Disponible en: http://wac. colostate.edu/books/wpww/, http://wac.colostate.edu/books/wpww/ chapter41.pdf

Carlino, P. (2013). Alfabetización académica diez años después. Revista Mexicana de Investigación Educativa, 18 (57), 355-381. Disponible en: http://www.redalyc.org/articulo.oa?id=14025774003 
Cassany, D. \& López-Ferrero, C. (2010). De la universidad al mundo laboral: continuidad y contraste entre las prácticas letradas académicas y profesionales. En Giovanni Parodi (ed.). Alfabetización académica y profesional en el siglo XXI. Leer y escribir desde las disciplinas, 347374. Santiago de Chile: Ariel.

Castelló, M.; Bañales-Faz, G. \& Vega-López, N. (2013). Leer múltiples documentos para escribir textos académicos en la universidad: o cómo aprender a leer y escribir en el lenguaje de las disciplinas. Revista Mexicana de Investigación Educativa, 18 (57), 461-481. Disponible en: http://www.scielo.br/scielo.php?script=sci_arttext\&pid $=$ S0103-73072011000100009

Ciapuscio, G. (2000). La monografía en la universidad: ¿una clase textual? Humanitas, Revista de la Facultad de Filosofía y Letras, Universidad Nacional de Tucumán, UNT, 23 (30-31), 227-253.

Cisneros, M.; Jiménez, H. \& Rojas, G. (2010). Alfabetización académica y profesional como directrices de la acción formativa en la educación superior. En Giovanni Parodi (ed.). Alfabetización académica y profesional en el siglo XXI. Leer y escribir desde las disciplinas, 291-315. Santiago de Chile: Ariel.

Cubo de Severino, L. (coord.) (2005). Los textos de la ciencia. Principales clases del discurso académico-científico. Córdoba: Editorial Comunicarte.

Ezcurra, A. M. (2011). Abandono estudiantil en educación superior. Hipótesis y conceptos. En Nora Gluz (ed.). Admisión a la universidad y selectividad social. Cuando la democratización es más que un problema de "ingresos", 23-62. Los Polvorines: Universidad Nacional de General Sarmiento, UNGS.

Gallardo, S. (2005). La monografía universitaria como aprendizaje para la producción de artículos científicos. En Graciela Vázquez (coord.). Español con fines académicos: de la comprensión a la producción de textos, 13-28. Madrid: Edinumen.

Gardner, S. \& Nesi, H. (2012). A Classification of Genre Families in University Student Writing. Applied Linguistics, 34 (1), 25-52. Manuscrito disponible en: https://curve.coventry.ac.uk/open/file/64b82bdd-f91d86f9-f661-cc472fbe523a/1/gardnercomb.pdf

Gardner, S. \& Powell, L. (2006). An Investigation of Genres of Assessed Writing in British Higher Education: A Warwick-Reading-Oxford Brookes Project. Paper presentado en el seminario Research, Scholarship and Practice in the Area of Academic Literacies, University of Westminster, 30 June. Disponible en http://www.wmin.ac.uk/ page-13087, http:// www2.warwick.ac.uk/fac/soc/al/research/collect/bawe/papers/

Gentile, F. \& Merlinsky, G. (2003). Perfil de los ingresantes al Primer Ciclo Universitario (PCU), Cohorte 2003. Informe inédito elaborado por la Secretaría Académica de la Universidad Nacional de General Sarmiento, UNGS.

Giudice, J.; Natale, L. \& Stagnaro, D. (2008). El proceso de construcción de un estudiante reflexivo: análisis evolutivo de un caso. Actas del III Congreso Internacional Transformaciones culturales. Debates de la teoría, la crítica y la lingüística. Buenos Aires: Facultad de Filosofía y Letras, Universidad de Buenos Aires.

González-Pinzón, B. Y. (2012). The Progressions and Transformations of the Program of Academic Reading and Writing (PLEA) in Colombia's Universidad Sergio Arboleda. En Chris Thaiss, Gerd Bräuer, Paula Carlino, Lisa Ganobcsik-Williams \& Aparna Sinha (eds.) (2012). Writing Programs Worldwide: Profiles of Academic Writing in Many Places, 157167. Anderson, South Carolina: Parlor Press. Disponible en: http:// 
wac.colostate.edu/books/wpww/, http://wac.colostate.edu/books/ wpww/chapter14.pdf

Halliday, M. A. K. (1978). El lenguaje como semiótica social. México: Fondo de Cultura Económica, FCE.

Hyland, K. (2000). Disciplinary Discourses: Social Interactions in Academic Writing. London: Longman.

Lea, M. R. \& Street, B. V. (1998). Student Writing in Higher Education: An Academic Literacies Approach. Studies in Higher Education, 23 (2), 157-172. Disponible en: https://www.kent.ac.uk/teaching/documents/ qualifications/studwritinginhe.pdf

Lea, M. R. \& Street, B. V. (2006). The 'Academic Literacies' Model: Theory and Applications. Theory into practice, 45 (4), 368-377. Disponible en: http://www.researchgate.net/publication/47343136_The_ academic_literacies_model_Theory_and_applications

Lewis, M. \& Starks, D. (1997). Revisiting Examination Questions in Tertiary Academic Writing. English for Specific Purposes, 16 (3), 197-210.

Lillis, T. (2003). Student Writing as 'Academic Literacies': Drawing on Bakhtin to Move from Critique to Design. Language and Education, 17 (3), 192-207. Disponible en: http://www.researchgate.net/ publication/42792331_Student_Writing_as_'Academic_Literacies' Drawing_on_Bakhtin_to_Move_from_Critique_to_Design

Lillis, T. \& Scott, M. (2007). Defining Academic Literacies Research: Issues of Epistemology, Ideology and Strategy. Journal of Applied Linguistics, 4 (1), 5-32.

MacDonald, S. P. (1994). Professional Academic Writing in the Humanities and Social Sciences. Carbondale, Illinois: Southern Illinois University Press.

Ministerio de Ciencia, Tecnología e Innovación Productiva (MinCyT) (2011). Argentina Innovadora 2020. Plan Nacional de Ciencia, Tecnología e Innovación Productiva de la Nación 2012-2015. Buenos Aires: MinCyT. Disponible en http://www.mincyt.gob.ar/adjuntos/archivos/ 000/022/0000022576.pdf

Molina-Natera, V. (2012). Escritura a través del currículo en Colombia: situación actual y desafíos. magis, Revista Internacional de Investigación en Educación, 5 (10), 93-108. Disponible en: http://www. redalyc.org/articulo.oa?id $=281024896006$

Montolío, E. \& López-Samaniego, A. (2010). Especificidades discursivas de los textos profesionales frente a los textos académicos: el caso de la recomendación profesional. En Giovanni Parodi (ed.). Alfabetización académica y profesional en el siglo XXI: Leer y escribir desde las disciplinas, 215-245. Madrid: Planeta.

Narvaja de Arnoux, E.; Alvarado, M.; Balmayor, E.; Stefano, M. di; Pereira, M. \& Silvestri, A. (1998). Talleres de lectura y escritura. Textos y actividades. Buenos Aires: Eudeba.

Narvaja de Arnoux, E.; Stefano M. di \& Pereira, C. (2002). La lectura y la escritura en la universidad. Buenos Aires: Eudeba.

Natale, L. (coord.) (2012). En carrera: escritura y lectura de géneros académicos y profesionales. Los Polvorines: Universidad Nacional de General Sarmiento, UNGS. Disponible en: http://www.ungs.edu.ar/cm/ uploaded_files/publicaciones/502_TB16\%20-\%20En\%20carrera\%20 2012\%20-\%20Web.pdf

Natale, L. (2013a). El semillero de la escritura. Las tareas escritas a lo largo de tres carreras de la UNGS. Los Polvorines: Universidad Nacional de General Sarmiento, UNGS. Disponible en http://www.ungs.edu.ar/areas/ publicaciones_temas/47/, http://www.ungs.edu.ar/cm/uploaded 
files/publicaciones/582_EDU14\%20El\%20semillero\%20de\%20 la\%20escritura_web.pdf

Natale, L. (2013b). Integración de enfoques en un programa institucional para el desarrollo de la escritura académica y profesional. Revista Mexicana de Investigación Educativa, 18 (58), 685-707. Disponible en: http://www.redalyc.org/articulo.oa?id=14027703002

Natale, L. \& Stagnaro, D. (2012). Desarrollo de habilidades de lectura y escritura en la trayectoria académica del ingeniero: la experiencia de un programa desafiante e innovador. Revista Argentina de Enseñanza de la Ingeniería, 13 (23), 45-52. Disponible en: http://www.ing.unrc. edu.ar/raei/archivos/img/arc_2013-03-17_20_52_15-N3T04.pdf

Natale, L. \& Stagnaro, D. (2015). Alfabetización profesional durante la carrera universitaria: entre la universidad y la empresa. Itinerarios Educativos, 7, 11-28.

Navarro, F. (ed.) (2014). Manual de escritura para carreras de humanidades. Buenos Aires: Editorial de la Facultad de Filosofía y Letras, Universidad de Buenos Aires, UBA.

Padilla, C. (2004). La comprensión de textos académicos en estudiantes universitarios: el caso de la dimensión polémica. RASAL, Revista de la Sociedad Argentina de Lingüística, 2, 45-66.

Padilla, C. (2012). Escritura y argumentación académica: trayectorias estudiantiles, factores docentes y contextuales. magis, Revista Internacional de Investigación en Educación, 5 (10), 31-57. Disponible en: http://www.redalyc.org/pdf/2810/281024896003.pdf

Padilla, C.; Douglas, S. \& López, E. (2007-2010). Yo expongo: taller de prácticas de comprensión y producción de textos expositivos. Córdoba: Comunicarte.

Parodi, G. (ed.) (2008). Géneros académicos y profesionales: accesos discursivos para saber hacer. Valparaíso: Ediciones Universitarias Valparaíso, EUV.

Parodi G. (ed.) (2010). Alfabetización académica y profesional en el siglo $X X I$. Leer y escribir desde las disciplinas. Santiago de Chile: Ariel.

Parodi, G.; Ibáñez, R.; Venegas, R. \& González, C. (2010). Identificación de géneros académicos y géneros profesionales: principios teóricos y propuesta metodológica. En Giovanni Parodi (ed.). Alfabetización académica y profesional en el siglo XXI. Leer y escribir desde las disciplinas, 249-289. Santiago de Chile: Ariel.

Pérez-Abril, M. \& Rodríguez-Manzano, A. (2013). ¿Para qué se lee y se escribe en la universidad colombiana? Caracterización de prácticas de lectura y escritura en 17 universidades. Revista de Docencia Universitaria, 11 (1), 137-160. Disponible en: http://red-u.net/redu/index. php/REDU/article/view/549

Riestra, D. (2002). Lectura y escritura en la universidad: las consignas de las tareas en la planificación de la reenseñanza de la lengua. RILL, Revista del Instituto de Investigaciones Lingüísticas y Literarias Hispanoamericanas, 15, 54-68.

Riestra, D. (2008). Las consignas de enseñanza de la lengua. Un análisis desde el interaccionismo socio-discursivo. Buenos Aires: Miño Dávila Editores.

Rinaudo, M.; Donolo, D. \& Paoloni, P. (2003). Tareas académicas en la universidad. Rol mediador de los planes cognitivos. En Memorias de la $X$ Jornadas de investigación Salud, Educación y Trabajo. Aportes de la investigación a la Psicología. Tomo I.

Rosales, P. \& Vázquez, A. (2006). Escribir y aprender en la universidad. Análisis de textos académicos de los estudiantes y de su relación con 
el cambio cognitivo. Revista Signo y Seña, 16, 47-69. Disponible en: http://www.escrituraylectura.com.ar/posgrado/revistas/SyS16.pdf

Rose, D. \& Martin, J. (2012). Learning to Write, Reading to Learn. Genre, Knowledge and Pedagogy in the Sydney School. Sheffield, United Kingdom: Equinox Publishing.

Russell, D. R. (1991). Writing in the Academic Disciplines, 1870-1990: A Curricular History. Carbondale, Illinois: Southern Illinois University Press.

Sabaj, O. (2009). Descubriendo algunos problemas en la redacción de Artículos de Investigación Científica (AIC) de alumnos de postgrado. Revista Signos, 42 (69), 107-127. Disponible en: http://www.scielo.cl/ pdf/signos/v42n69/a06.pdf

Secretaría de Políticas Universitarias, SPU (2010). Anuario de estadísticas universitarias. Buenos Aires: Secretaría de Políticas Universitarias, SPU. Disponible en: http://portales.educacion.gov.ar/spu/investigacion-y-estadisticas/anuarios/, http://repositorio.educacion.gov. ar:8080/dspace/bitstream/handle/123456789/109591/Anuario_de_ Estadisticas_Universitarias_2010.pdf?sequence $=1$

Stagnaro, D. \& Martínez, D. (2013). Prácticas de formación docente: exploración de los instrumentos de evaluación. Actas de las VII Jornadas sobre Formación del Profesorado. Mar del Plata: Universidad Nacional de Mar del Plata.

Stagnaro, D. \& Navarro, F. (2013). Consignas de evaluación en la carrera de Ingeniería Industrial: articulaciones entre la formación universitaria y la inserción profesional. En Lucía Natale (coord.). El semillero de la escritura. Las tareas escritas a lo largo de tres carreras de la UNGS. Los Polvorines: Universidad Nacional de General Sarmiento, UNGS. Disponible en http://www.ungs.edu.ar/areas/publicaciones_temas/47/, http://www.ungs.edu.ar/cm/uploaded_files/publicaciones/582 EDU14\%20El\%20semillero\%20de\%20la\%20escritura_web.pdf

Swales, J. (1990). Genre Analysis: English in Academic and Research Settings. Glasgow: Cambridge University Press. 